\title{
The Effect of Consultations on the Cost of Pre-Anesthetic Assessment
}

\author{
Omer L. Erhan ${ }^{1}$, Azize Bestas ${ }^{1,2 *}$, Sait F. Oner ${ }^{1}$, Turkay Yucel $^{1}$, Sibel Ozcan $^{1}$, Hasan Arkk ${ }^{1}$ \\ ${ }^{1}$ Department of Anesthesiology and Reanimation, Firat University School of Medicine, Elazı $\breve{g}$, Turkey; ${ }^{2}$ Department of Anesthesiol- \\ ogy and Reanimation, Firat University School of Medicine, Elazı $\breve{g}$, Turkey.

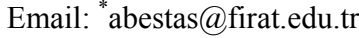

Received September $17^{\text {th }}, 2013$; revised October $15^{\text {th }}, 2013$; accepted November $3^{\text {rd }}, 2013$

Copyright (C) 2013 Omer L. Erhan et al. This is an open access article distributed under the Creative Commons Attribution License, which permits unrestricted use, distribution, and reproduction in any medium, provided the original work is properly cited.

\begin{abstract}
Background: In our study, researching the distribution of the consultations other than anaesthesia in compliance with the disciplines and ages of the patients and its effect on the assessment cost has been aimed. Methods: Our study has been conducted during 8 months by the retrospective assessment of the records of 3844 cases on which an elective surgical procedure was planned in the anesthesiology polyclinic of Firat University Hospital. The records of patients have been obtained through examining their files from the hospital record system. Results: The total number of patients whose consultations have been demanded was 367 (9.5\%) and the number of male/female was 192/175. The number of cases about which more than one consultation has been demanded was determined as $87(2.2 \%)$. The number of cases on which a method change for the anaesthesia was applied was $21(5.7 \%)$, and the number of cases whose surgery was cancelled was $3(0.81 \%)$. Requested consultation for each patient, the consultations of cardiology, chest diseases and internal medicine bring an additional cost on the total sum respectively as 37.2 TL (28.61 \$), $29.8 \mathrm{TL}$ (22.92 \$) and 19.9 TL (15.30 \$). Conclusions: We suggest that the cost analysis should be absolutely taken into consideration during the pre-anaesthetic assessment for the requirement of routine examinations.
\end{abstract}

Keywords: Pre-Anaesthetic Asessment; Consultations; Costs

\section{Introduction}

Pre-operative assessment is significant because of the facts that the planned-surgery may be cancelled or this assessment may prevent the cancellation of the surgery or the period of hospitalization may be shortened and moreover anaesthesia should be performed under the safe conditions. This assessment, by determining health problems which are not detected before, enables the revision of the anaesthetic and surgical approaches and the prevention of undesirable events during the preoperative and post-operative period. For this purpose, during the preoperative period, many laboratory tests and examinations are conducted on patients [1]. The consultations other than anaesthesia compose some part of this assessment. These consultations are applied because accompanied diseases bring additional costs on the routine assessment.

In our study, researching the distribution of the consultations other than anaesthesia in compliance with the disciplines and age groups and its effect on the asse${ }^{*}$ Corresponding author. ssment cost has been aimed.

\section{Materials and Methods}

Our study has been conducted during 8 months by the retrospective assessment of the records of 3844 cases on which an elective surgical procedure was planned in the anesthesiology polyclinic of Firat University Hospital. The records of patients have been obtained through examining their files from the hospital record system. Preoperative tests for the age group of under 15 years, complete blood count, fixing of blood group, blood glucose level, aspartate transaminase, alanine transaminase, urinalysis, activated partial thromboplastin time and prothrombin time have been accepted as routine costs. Electrocardiogram, for the patients more than 35 years old and postero-anterior chest X-ray and renal function tests for those who are more than 65 years old are added to these tests and the routine cost has been found. For each patient for whom the consultation has been demanded the name, surname, protocol, age (pediatric age $<15$ years, 
adult age 15 to 65 years and geriatric age $>65$ years), weight, gender, information about from which service of the hospital she/he comes, his/her planned-operation, the reason of the consultation demanded, additional tests demanded as a result of the consultation, and the changes on the anaesthesia technique have been recorded. It has been found that in compliance with the age groups and related disciplines (Cardiology, Chest Diseases, Internal Medicine), the cases for which consultation is demanded by considering pre-operative pathology bring an additional cost to the total sum of the consultation.

For the calculation of the costs, the prices fixed and applied for the year of study by the Precept of Budget Practice (PBP) of the Ministry of Finance have been taken into consideration. Based on the value of the time, the cost has been also fixed as USA dollar (\$) by considering $1.3 \mathrm{TL}=1 \$ \mathrm{TL}$.

\section{Results}

When the findings of 3844 patients have been assessed, the total number of patients whose consultations have been demanded were recorded as $367(9.5 \%)$ and the number of male/female was found as $192 / 175$. When examined in compliance with the age groups, the consultation has been demanded for 8 patients $(2.1 \%)$ who are under 15 years old, 228 patients $(62.1 \%)$ who are between 15 to 65 years old and 131 patients (35.6\%) who are more than 65 years old. The number of cases about which more than one consultation has been demanded was fixed as $87(2.2 \%)$. The number of cases on which a method change for the anaesthesia was applied was 21 $(5.7 \%)$, the number of cases whose surgery was cancelled was $3(0.81 \%)$ and the number of cases which were diagnosed for the first time is 104 .

The cost occured as a result of the consultation has been recorded in compliance with the disciplines and age groups (Table 1). It has been determined as routine cost, additional cost and total cost in compliance with the age groups (Table 2). When assessed alone, requested consultation for each, patient the consultations of cardiology, chest diseases and internal medicine bring an additional cost on the total sum respectively as 37.2 TL (28.61 \$), $29.8 \mathrm{TL}(22.92 \$)$ and $19.9 \mathrm{TL}(15.30 \$)$.

The cost has been found as 62,54 TL (48.10 \$)/per patient for those the cardiology and chest diseases consultation were demanded 59,07 TL (45.43 \$)/per patient for those internal medicine and chest diseases consultation were demanded and 43.44 TL (33.41 \$)/per patient for those the cardiology and internal medicine consultation were demanded.

\section{Discussion}

During the pre-operative assessment, anamnesis, physical examination and the choice of examination should be in
Table 1. Units from which consultations have been demanded and the cost. TL: Turkish lira, \$: USA dollar.

\begin{tabular}{cccc}
\hline & Cardiology & Chest Diseases & $\begin{array}{c}\text { Internal } \\
\text { Medicine }\end{array}$ \\
\hline $\begin{array}{c}\text { Consultation } \\
\text { [n(\%)] }\end{array}$ & 154 & 45 & 294 \\
Total Cost & & $(1.17)$ & $(7.64)$ \\
• TL & 5298.10 & & \\
- $\quad \mathbf{\$}$ & 4075.46 & 877.23 & 735.19 \\
\hline
\end{tabular}

Table 2. The routine and additional costs [Turkish lira (TL), USA dollar (\$)] in compliance with the age groups.

\begin{tabular}{cccc}
\hline \multirow{2}{*}{ Age (years) } & \multicolumn{3}{c}{ Cost TL (USA \$) } \\
\cline { 2 - 4 } & Routine cost & Additional cost & Total cost \\
\hline & & & \\
$<\mathbf{1 5}$ & $26.34(20.26)$ & $18.2(14.00)$ & $44.54(34.26)$ \\
$\mathbf{1 5}$ to 65 & $29.34(22.56)$ & $29.60(22.76)$ & $58.94(45.33)$ \\
$>\mathbf{6 5}$ & $42.74(32.87)$ & $36.78(28.29)$ & $79.52(61.16)$ \\
& & & \\
\hline
\end{tabular}

connection with the determination of ambigious cases which have the potential to affect the pre-operative results [2]. Making the pre-operative assessment by individualizing it toward the patient, taking the anamnesis attentively and making a detailed physical examination may reduce the demand for consultation other than anaesthesia and thus cost. Unnecessary tests and scrutinies lead hospital service load and cost to increase [2]. Fischer et al. [3] determined that preventing unnecessary tests may reduce the cost by $55 \%$.

It is known that making the pre-anesthetic assessment before the operation day prevents last minute cancellations and postponing the surgical operation [4]. Besides surgery costs related to postponing the case increases, using the operation room effectively is not possible as the surgery date can not be clarified. It is reported that a monetary loss of 1.500 USD per hour for cancellation of the surgery and 10 USD per minute for postponing the surgery occurs [5].

Ferrando et al. [6] have found the cost for pre-operative assessment cost as $6.10 €$ within the age group of $<15$ years old, $27.16 €$ within the age group of $15-65$ years old, and $38.48 €$ within the age group of $>65$ years old. The results of this study are similar to ours in terms of the increase in cost. Gold et al. [7] claimed that abnormal findings are significantly high within those who are more than 60 .

The results of our study are significant because of the fact that the pre-anaesthetic assessment has revealed different costs in compliance with the age groups. As a result of our assessment, 9.5\% of our patients have been applied the consultations other than anaesthesia.

\section{Conclusion}

As one becomes older, the proportion of consultation and 
the cost during the pre-anaesthetic assessment rise parallelly to the age. We suggest that the results of our study are taken into consideration within the framework of the health policies in terms of revealing different costs in compliance with the age groups during the preparation of package programmes while PBP is being prepared. Therefore, the cost analysis should be absolutely taken into consideration during the pre-anaesthetic assessment for the requirement of routine examinations.

\section{REFERENCES}

[1] N. Imasogie, D. T. Wong, K. Luk and Chung F, "Elimination of Routine Testing in Patients Undergoing Cataract Surgery Allows Substantial Saving in Laboratory Costs. A Brief Report," Canadian Journal of Anesthesia, Vol. 50, No. 3, 2003, pp. 246-248.

[2] B. A. Finegan, S. Rashiq, F. A. McAlister and P. O'Connor, "Selective Ordering of Preoperative Investigations by Anesthesiologist Reduces the Number and Cost of Tests," Canadian Journal of Anesthesia, Vol. 52, No. 6, 2005, pp. 575-580.

[3] S. P. Fischer, "Development and Effectiveness of an Anesthesia Preoperative Evaluation Clinic in a Teaching
Hospital," Anesthesiology, Vol. 85, No. 1, 1996, pp. 196206.

http://dx.doi.org/10.1097/00000542-199607000-00025

[4] C. M. Haberkern and J. H. Lecky, "Preoperative Assessment and the Anesthesia Clinic," Anesthesiology Clinics of North America, Vol. 14, No. 4, 1996, pp. 609-630. http://dx.doi.org/10.1016/S0889-8537(05)70297-2

[5] D. J. Corell, A. M. Bader, M. W. Hull, C. Hsu, L. C. Tsen, and D. L. Hepner, "Value of Preoperative Clinic Visits in Identifying Issues with Potential Impact on Operating Room Efficiency," Anesthesiology, Vol. 105, No. 6, 2006, pp. 1254-1259. http://dx.doi.org/10.1097/00000542-200612000-00026

[6] A. Ferrando, C. Ivaldi, A. Buttiglieri, E. Pagano, C. Bonetto, R. Arione, L. Scaqlione, E. Gelormino, F. Merletti and G. Ciccone, "Guidelines for Preoperative Assessment: Impact on Clinical Practice and Costs," International Journal of Quality in Health Care, Vol. 17, No. 4, 2005, pp. 323-329. http://dx.doi.org/10.1093/intqhe/mzi039

[7] B. S. Gold, M. L. Young, J. L. Kinman, D. S. Kitz, J. Berlin and J. S. Schwartz, "The Utility of Preoperative Electrocardiograms in the Ambulatory Surgical Patient," Archives of Internal Medicine, Vol. 152, No. 2, 1992, pp. 301-305.

http://dx.doi.org/10.1001/archinte.1992.00400140055013. 